Alternatively, PAF can be estimated if the incidence of the disease in the population, and the incidence of the disease in the unexposed, are known. Improved disease surveillance can make an important contribution in areas where exposure prevalence measures are not good and difficult to improve. Good estimates of disease that can reliably be connected to work (e.g. occupational asthma, occupational dermatitis) in specific study populations would allow the estimate of incidence in the unexposed if the incidence in the population was known. This approach would be more difficult in circumstances where the direct connexion to work is harder to establish (e.g. many musculoskeletal disorders and noise-induced hearing loss). Alternatively, since estimates of the incidence of the disease in the general population will often be available, probably the key area of additional information required would actually be the incidence in unexposed persons. This isn't the typical focus of occupational disease surveillance but could provide a useful contribution to the understanding of occupational disease and its burden on the community.

Thirdly, surveillance systems that cover disorders that are virtually uniquely occupational, such as a register of cases of malignant mesothelioma, can provide a direct estimate of the number of cases of a particular occupational disorder.

Conclusions Occupational surveillance systems can potentially contribute to estimations of burden of disease studies using several different approaches.

\section{HOW USING GEOGRAPHICAL INFORMATION SYSTEMS (GIS) COULD ALLOW US TO IMPROVE OCCUPATIONAL DISEASES (OD) SURVEILLANCE?}

${ }^{1} \mathrm{M}$ D Delaunay, ${ }^{2}$ Godard, ${ }^{3}$ Bicout, ${ }^{4}$ de Gaudemaris, ${ }^{4}$ Bonneterre. ${ }^{1}$ Paris, France; ${ }^{2}$ UMR CNRS 3258, Maison des sciences de l'homme (MSH), Paris Nord, Saint-Denis, La Plaine, France; ${ }^{3}$ UJF-Grenoble 1/CNRS/TIMC-IMAG UMR 5525 (EPSP team), Grenoble, France; ${ }^{4}$ Grenoble Teaching Hospital : Occupational and Environmental Diseases Centre, Grenoble, France

\subsection{6/oemed-2013-101717.201}

Objective To show and illustrate to what extent, GIS could be an opportunity for work-related and occupational diseases surveillance, in combining relevant "spatialisable" information from different data sources, in order to help analyse and present existing data in a systematic, and easily understandable way; which might be of great help for surveillance, vigilance, and prevention purposes.

Methods Cases of Work related diseases ("numerator") from different and complementary sources, as well as data related to underlying industrial tissue and working population according to activity sectors ("denominator") are to be georeferenced and projected (mapped together) with GIS software. For the purpose of our illustration, numerator data are those from the French National Surveillance Scheme on OD Surveillance and Prevention rnv3p, and data from a compensated scheme; data from the denominator are files of enterprises from Chambers of Commerce and Industry as well as from the French National Health insurance company for salaried workers.

Supplementary qualitative knowledge might also be gathered, georeferenced and mapped to increase the level of information produced (here: data from "on the ground" by occupational physicians). We used ArcGIS ( $9^{\text {th }}$ and $10^{\text {th }}$ version) software and basemaps from the National Geographic Institute (IGN) and OpenStreetMap (OSM).

Results Results are illustrated by maps derived from analyses concerning one specific activity sector. The differences and complementarities in case capture between the work-related diseases surveillance scheme and the compensated OD register are highlighted. The spreading of some toxicological risks from companies to their subcontractors is also shown.

Conclusion This methodology, by combining different data sources and a convenient visualisation of the results, is of a great help to adopt a systemic and integrate point of view on OD. Furthermore, it might help surveillance systems to better analyse some issues of concern (capture, shading zone, geographical patterns of referrals or reporting, etc[3DOTS]).

Acknowledgements rnv3p, ANSES, CNAM-TS.

\section{INFORMING PUBLIC POLICY IN OCCUPATIONAL HEALTH THROUGH DISEASE SURVEILLANCE DATA - THE 'THOR' EXAMPLE. (MINISYMPOSIUM: OCCUPATIONAL DISEASE SURVEILLANCE)}

R M Agius. University of Manchester, Manchester, United Kingdom

10.1136/oemed-2013-101717.202

Objectives To discuss how voluntary occupational health surveillance can help informing public policy in relation to health at work.

Methods and Results The Health and Occupation Research Network (THOR) collects incidence data on occupational disease and work related ill health (WRI).

Incidence data from THOR has been used to inform and direct UK policy and priorities in relation to occupational asthma and dermatitis such as in bakers and spray painters. THOR played an important part in measuring the UK's "Revitalising Health and Safety" targets: It was the main UK data source used to report the statistically significant decrease in both work-related asthma and skin disease in the decade after 2009. The effects of interventions, such as implementation of statute, relating to chromate, latex, and glutaraldehyde were studied using incidence data and demonstrated the value of these public policy initiatives. 'Before and after' comparisons were made to investigate changes in sickness absence certification following the introduction of the 2010 'fitnote' which assisted GPs to recommend workplace adjustments. In the first year the new category certified as 'fit' with recommended adjustments was almost exclusively limited to that proportion of workers previously certified as 'fit'. However after the first year there was evidence that the GPs were also making rehabilitation recommendations in those who would previously have been certified as 'sick'.

A collaborative international consortium entitled MODERNET (Monitoring trends in Occupational Diseases and tracing new and Emerging Risks in a Network) has been established with EU funding. MODERNET is developing improvements in data quality, trends analysis and disease vigilance, intended to influence public policy at a supra-national level.

Conclusions Data from voluntary occupational health surveillance schemes are valuable in informing public policy for example by monitoring trends in WRI incidence, and in evaluating the benefits of interventions.

203

INTERVENTIONS FOR REDUCING THE UNDERREPORTING OF OCCUPATIONAL DISEASES - MINISYMPOSIUM 'IMPROVING THE IMPACT OF OCCUPATIONAL DISEASE SURVEILLANCE' 